\title{
Preface: Radio Heliophysics: Science and Forecasting
}

\author{
Mario M. Bisi ${ }^{1}$ - J. Americo Gonzalez-Esparza ${ }^{2}$. \\ Bernard V. Jackson ${ }^{3} \cdot$ Munetoshi Tokumaru ${ }^{4}$. \\ John Leibacher ${ }^{5}$
}

Published online: 20 October 2015

(C) Springer Science+Business Media Dordrecht 2015

\section{Introduction}

Heliophysics is considered to be the all-encompassing sub-field of astrophysics that includes subjects from the Sun's core to planetary cores, and indeed, to the edge of the heliosphere where the Sun's influence in the Galaxy ends and that of the interstellar medium (IsM) takes over. There are many different physical domains within heliophysics, where the physical specifications and boundary conditions vary considerably, and thus it is a wide area of

Radio Heliophysics: Science and Forecasting

Guest Editors: Mario M. Bisi, Bernard V. Jackson, and J. Americo Gonzalez-Esparza

M.M. Bisi

mario.bisi@stfc.ac.uk

J. Americo Gonzalez-Esparza

americo@geofisica.unam.mx

B.V. Jackson

bvjackson@ucsd.edu

M. Tokumaru

tokumaru@nagoya-u.ac.uk

J. Leibacher

john.leibacher.sola@gmail.com

1 RAL Space, Science \& Technology Facilities Council - Rutherford Appleton Laboratory, Harwell Oxford, Oxfordshire, OX11 0QX, England, UK

2 Instituto de Geofisica, Unidad Michoacan, Universidad Nacional Autonoma de Mexico, Mexico City, Mexico

3 Center for Astrophysics and Space Sciences, University of California, 9500 Gilman Drive \#0424, La Jolla, San Diego, CA 92093-0424, USA

4 Solar-Terrestrial Environment Laboratory, Nagoya University, Furo-Cho, Chikusa-ku, Nagoya 464-8601, Japan

5 National Solar Observatory, Tucson, AZ, USA 
current scientific research. Radio heliophysics is the part of heliophysics where radio observations, measurements, emission, and detections play a role in our overall understanding of the heliophysical system.

Contributions for this Topical Issue (TI) of Solar Physics include articles from many aspects of radio heliophysics where radio (observations, measurements, data, results, modeling, etc.) forms the basis of each article or is a strong complementary data set to other heliophysics observations, measurements, or modelling. The aim of this TI is to highlight the wide uses, scope, and, indeed, capabilities and power of radio observations in scientific and space-weather forecasting investigations of our solar system and the Sun's influence from its core to the heliosphere-IsM boundary and especially upon the Earth's environment. Observations and modelling of the heliosphere through or by incorporating radio methods and radio data are of a critical importance to improving our understanding of the physics behind the various components of the heliophysical system and how each of these components ties into the next; these are discussed and highlighted in the various articles. Radio observations are also essential to advance our understanding of space weather both in the vicinity of Earth and at other solar-system bodies (e.g. the Sun-Earth connection in its many facets from the solar dynamo to ground-level events at Earth's surface).

\section{Workshop}

This TI was stimulated by the interplanetary scintillation (IPS) workshop that took place at Nagoya University (following the CAWSES-II meeting) hosted by the Solar-Terrestrial Environment Laboratory (STEL), Japan, in November 2013, and much of this TI is from work and ideas discussed at and following the workshop.

The workshop SOC was comprised of three of the authors of this TI Preface: Munetoshi Tokumaru, who also served as the LOC, Mario M. Bisi, and Bernard V. Jackson. That workshop was the seventh in a series of IPS, Radio-Heliophysics, and Remote-Sensing workshops held between 2004 and 2013. Financial support for the workshop was provided from the JSPS Grand-in-Aid for Scientific Research-A 25247079 (M. Tokumaru) and the Korean Space Weather fund to USCD (B.V. Jackson). Twenty-three researchers participated in the workshop and discussed analytical methods and the standardization of IPS data as well as the results of their studies and their future plans; further details of this workshop series have been given by Bisi et al. (2013); see also stsw1.stelab.nagoya-u.ac.jp/ips_nagoya.html). One major outcome of this workshop was the initial definition of a standardized IPS data set, which is due to be officially released as the IPS Common Data Format v1.0 (IPSCDFv1.0) in 2015. This will provide non-IPS specialists with a better way to ascertain, access, and use some basic IPS results and data products.

\section{Summary}

The next workshop, the eighth in the series overall, is entitled "Third Remote Sensing of the Inner Heliosphere and Space Weather Applications Workshop", taking place in Morelia, Mexico, 20-24 November 2015. The core organizers are three of the authors of this TI Preface, J. Americo Gonzalez-Esparza, Mario M. Bisi, and Bernard V. Jackson, as well as David F. Webb from Boston College, MA, USA. Following this workshop, IPSCDFv1.0 is expected to be released. 
Finally, we thank the many authors for the influx of extra papers from those not able to attend the IPS Workshop in 2013 as well as the articles from those in attendance. We also convey our special thanks to the team of referees, who worked diligently in making sure the articles contained within this TI of Solar Physics on "Radio Heliophysics: Science and Forecasting" are of the highest scientific quality and content. We are pleased with the articles in this TI and are very happy that they collectively cover many aspects of radio heliophysics techniques, observations, modelling, data analyses, and comparisons with other forms of data and modelling from near the Sun's "surface" out to beyond the Earth, including the influence on the Earth's space environment.

\section{Articles}

Bisi, M.M., Gonzalez-Esparza, J.A. Jackson, B.V., Tokumaru, M., Leibacher, J.: 2015, Preface: Radio Heliophysics: Science and Forecasting. Solar Phys. 290, 2393, DOI.

Efimov, A.I., Lukanina, L.A., Rogashkova, A.I., Samoznaev, L.N., Chashei, I.V., Bird, M.K., Pätzold, M.: 2015, Coronal radio occultation experiments with the helios solar probes: correlation/spectral analysis of faraday rotation fluctuations. Solar Phys. 290, 2397, DOI.

Kishore, P., Ramesh, R., Kathiravan, C., Rajalingam, M.: 2015, A low-frequency radio spectropolarimeter for observations of the solar corona. Solar Phys. 290, 2409, DOI.

Tun Beltran, S.D., Cutchin, S., White, S.: 2015, A new look at type-III bursts and their use as coronal diagnostics. Solar Phys. 290, 2423, DOI.

Corona-Romero, P., Gonzalez-Esparza, J.A., Aguilar-Rodriguez, E., De-la-Luz, V., MejiaAmbriz, J.C.: 2015, Kinematics of ICMEs/shocks: blast wave reconstruction using type-II emissions. Solar Phys. 290, 2439, DOI.

Cremades, H., Iglesias, F.A., St. Cyr, O.C., Xie, H., Kaiser, M.L., Gopalswamy, N.: 2015, Low-frequency type-II radio detections and coronagraph data to describe and forecast the propagation of 71 CMEs/shocks. Solar Phys. 290, 2455, DOI.

Hariharan, K., Ramesh, R., Kathiravan, C.: 2015, Observations of near-simultaneous splitband solar type-II radio bursts at low frequencies. Solar Phys. 290, 2479, DOI.

Fujiki, K., Tokumaru, M., Iju, T., Hakamada, K., Kojima, M.: 2015, Relationship between solar-wind speed and coronal magnetic-field properties. Solar Phys. 290, 2491, DOI.

Aguilar-Rodriguez, E., Mejia-Ambriz, J.C., Jackson, B.V., Buffington, A., RomeroHernandez, E., Gonzalez-Esparza, J.A., Rodriguez-Martinez, M., Hick, P., Tokumaru, M., Manoharan, P.K.: 2015, Comparison of solar wind speeds using wavelet transform and fourier analysis in IPS data. Solar Phys. 290, 2507, DOI.

Yu, H., Jackson, B.V., Hick, P.P., Buffington, A., Odstrcil, D., Wu, C., Davies, J.A., Bisi, M.M., Tokumaru, M.: 2015, 3D Reconstruction of Interplanetary Scintillation (IPS) remotesensing data: global solar wind boundaries for driving 3D-MHD models. Solar Phys. 290, 2519, DOI. 
Mejia-Ambriz, J.C., Jackson, B.V., Gonzalez-Esparza, J.A., Buffington, A., Tokumaru, M., Aguilar-Rodriguez, E.: 2015, Remote-sensing of solar wind speeds from IPS observations at 140 and $327 \mathrm{MHz}$ using MEXART and STEL. Solar Phys. 290, 2539, DOI.

Romero-Hernandez, E., Gonzalez-Esparza, J.A., Aguilar-Rodriguez, E., OntiverosHernandez, V., Villanueva-Hernandez, P.: 2015, Detection of solar wind disturbances: Mexican array radio telescope IPS observations at $140 \mathrm{MHz}$. Solar Phys. 290, 2553, DOI.

Aguilar-Rodriguez, E., Tyul'bashev, S.A., Chashei, I.V., Romero-Hernandez, E.: 2015, Interplanetary Scintiallation (IPS) of the radio source 3C48 during periods of low and high solar activity. Solar Phys. 290, 2567, DOI.

Chashei, I.V., Shishov, V.I., Tyul'bashev, S.A., Subaev, I.A., Oreshko, V.V., Logvinenko, S.V.: 2015, Global structure of the turbulent solar wind during 24 solar activity maxima from IPS observations with the multibeam radio telescope BSA LPI at $111 \mathrm{MHz}$. Solar Phys. 290, 2577, DOI.

Sokół, J.M., Swaczyna, P., Bzowski, M, ,Tokumaru, M.: Reconstruction of Heliolatitudinal Structure of the Solar Wind Proton Speed and Density. Solar Phys. 290, 2589, DOI

Acknowledgements It is a pleasure to acknowledge all of the editors and staff at Solar Physics and Springer for their work in pulling this TI together, and thanks again to the team of referees for their time and effort in making this a very successful TI that covers a broad range of topics and studies related to radio heliophysics.

We would also like to thank STELab at Nagoya University for providing the rooms and internet access for the Workshop as well as for travel funding for several of the participants. We acknowledge UCSD funding received from the Korean Space Weather Center (KSWC) that provided lunches, coffee breaks, travel, and subsistence during the workshop, and for discussions following for some participants. In addition, we thank SCOSTEP CAWSES II for funding several workshop participants to the CAWSES-II meeting, which enabled them to subsequently stay on for the IPS Workshop.

Disclosure of Potential Conflicts of Interest The authors declare that they have no convicts of interest.

\section{References}

Bisi, M.M., Harrison, R.A., Lugaz, N., van Driel-Gesztelyi, L., Mandrini, C.H.: 2013, Solar Phys. $285,1$. DOI. 